\title{
Identification of novel splicing variants from RON proto-oncogene pre-mRNA
}

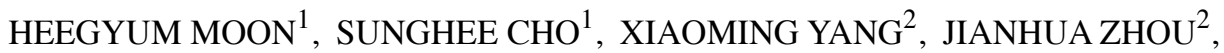 \\ TIING JEN LOH ${ }^{1}$, XUEXIU ZHENG ${ }^{1}$ and HAIHONG SHEN ${ }^{1}$ \\ ${ }^{1}$ School of Life Sciences, Gwangju Institute of Science and Technology, Gwangju 500-712, Republic of Korea; \\ ${ }^{2}$ JiangSu Key Laboratory of Neuroregeneration, Nantong University, Nantong, P.R. China
}

Received May 25, 2012; Accepted July 16, 2012

DOI: $10.3892 /$ or.2012.2043

\begin{abstract}
RON is a proto-oncogene that induces cell dissociation, migration and matrix invasion. $\mathrm{RON} \Delta 160$, a splicing variant of RON, is a natural splicing product in colon cancers that is produced through skipping of exons 5 and 6 in alternative splicing process. RON $\Delta 160$ promotes cellular transformation in vitro and tumor formation in vivo. We present, here, two novel splicing variants of RON in the partial splicing events that involve exons 5 and 6. The common facts of these two novel splicing variants are that exons 4-7 are included. In addition, intron 4 is spliced whereas intron 5 is included in both variants. The difference of these two isoforms is the inclusion or skipping of intron 6 . In one variant intron 6 is included, but intron 6 is skipped in another variant. These two variants should be truncated but these proteins have not yet been detected.
\end{abstract}

\section{Introduction}

RON receptor tyrosine kinase is a member of the MET protooncogene that induces cell dissociation, migration, and matrix invasion (1-3). Receptor tyrosine kinases consist of a large group of cell surface proteins with unique structure and biological activities (4,5). Abnormal accumulation and activation of receptor tyrosine kinases causes the initiation and progression of a variety of malignancies including tumors derived from breast and kidneys $(6,7)$. The invasive growth features are controlled by a genetic program which is conserved in MET family members $(8,9)$. Mature RON is a $180-\mathrm{kDa}$ heterodimeric protein that is composed of a $40-\mathrm{kDa}$ extracellular $\alpha$ chain and $150 \mathrm{kDa}$ transmembrane $\beta$ chain with intrinsic protein tyrosine kinase activity $(10,11) . \alpha$ and $\beta$ chains are proteolytic products of a $180-\mathrm{kDa}$ RON precursor (12). RON is activated by macrophage-stimulating protein (MSP), a serum protein that

Correspondence to: Dr Haihong Shen, School of Life Sciences, Gwangju Institute of Science and Technology, Gwangju 500-712, Republic of Korea

E-mail: haihongshen@gist.ac.kr

Key words: RON, splicing, variants, exon, intron is constitutively expressed by liver cells as an inactive form and requires proteolytis conversion for receptor binding (13-15). The binding of MSP to RON results in receptor autophosphorylation and upregulation of RON kinase activity, which in turn stimulates a number of intracellular pathways mediating MSP effects (16). In normal cells MSP-induced activation is a transient event, whereas in tumor cells RON activity is often constitutively upregulated (17). RON activation causes invasive growth and motility of certain epithelial tumor cells $(18,19)$. A number of facts suggest that RON might be involved in the progression of certain epithelial malignancies, particularly at the stage of tumor metastasis (20).

It has been shown that receptor tyrosine kinases can be activated by a variety of mechanisms, including mutation, deletion, gene rearrangement and alternative splicing of pre-mRNA (21). Various RON protein isoforms are produced through alternative splicing of pre-mRNA (12). One of the protein variants is Ron $\Delta 160$, a naturally occurring oncogenic form of RON identified in human colon cancers, which is produced from a splicing mRNA transcript by exons 5 and 6 . Ron $\Delta 160$ causes structural changes that lead to cellular transformation in vitro and tumor growth in vivo $(22,23)$. Another variant Ron $\Delta 170$ is a $170-\mathrm{kDa}$ variant is generated by skipping of exon 19 in alternative splicing (24). Ron $\Delta 170$ is kinase defective and acts as dominant negative agent that inhibits tumorigenic activities mediated by oncogenic variant RON $\Delta 160$ in colon cancer cells (24). Ron $\Delta 165$ is produced by an in-frame deletion of exon 11 that affects the proteolytic process which results in the accumulation of single-chain pro-Ron $\Delta 165$ in the cytoplasm (25). Ron $\Delta 155$ is a derived from a combined deletion of exons 5, 6 and 11 , the function of $\operatorname{Ron} \Delta 155$ is similar to $\operatorname{Ron} \Delta 160(25,26)$.

Here we show two novel splicing variants of RON protooncogene. From the sequence analysis it is proved they are partially spliced during the splicing events of exons 5 and 6 . These two variants contain either intron 5 , or introns 5 and 6 , in addition to exons 4-7. The proteins encoded by these variants are not identified yet.

\section{Materials and methods}

Cell culture. HeLa and C33A cells were maintained in Dulbecco's modified Eagle's medium (DMEM) and HCT116 and HT-29 cells were maintained in RPMI-1640 medium supplemented 


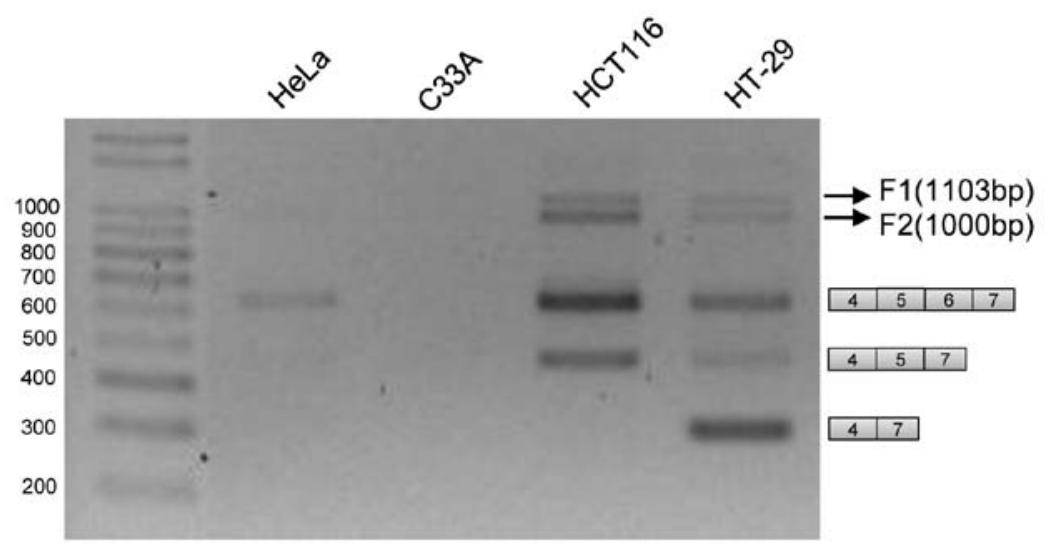

Figure 1. Alternative splicing of endogenous RON pre-mRNA. RT-PCR products of RON mRNA using a RON exon 4-specific forward primer and an exon 7-specific reverse primer are shown.

A.

$<\mathrm{F} 1$ sequence $>-1103 \mathrm{bp}$

GTTTTCCAGGTACCTATCCAAGGCCCTGGCTGCCGCCACTTCCTGACCTGTGGGCGTT GCCTAAGGGCATGGCATTTCATGGGCTGTGGCTGGTGTGGGAACATGTGCGGCCAGC AGAAGGAGTGTCCTGGCTCCTGGCAACAGGACCACTGCCCACCTAAGCTTACTGAGTT CCACCCCCACAGTGGACCTCTAAGGGGCAGTACAAGGCTGACCCTGTGTGGCTCCAA CTTCTACCTTCACCCTTCTGGTCTGGTGCCTGAGGGAACCCATCAGGTCACTGTGGGC CAAAGTCCCTGCCGGCCACTGCCCAAGGACAGCTCAAAACTCAGgtacaatctggtccctcccct ccctttccctgaagggggaaaccaagcagccccttccccatgagaccctgttctctgcttatcagaggcaaggggggatggggg aagctgcagtggttctgactgcttttgagagtcaaaaaggtctctgtcccttttgagctttcatgtgcccttcctgtctgttcactcatgg accagccaaggttaatctctgccccaccagaaccttccttccatggagggaggcatgggtggagaaatgecattctctggetcag aggagccctgtggcttgtggcagg atg atctaggcctgtgtaattcctggctgacctcaggggttcccctggtgccccagACC AGTGCCCCGGAAAGACTTTGTAGAGGAGTTTGAGTGTGAACTGGAGCCCTTGGGCAC CCAGGCAGTGGGGCCTACCAACGTCAGCCTCACCGTGACTAACATGCCACCGGGCAA GCACTTCCGGGTAGACGGCACCTCCGTGCTGAGAGGCTTCTCTTTCATGgtgaggctacctt gccctgtctgtgccettggccagtgcatggtacgggaagggaggggettggagtggaggacctgcctaagccacctctatgtcct cttagGAGCCAGTGCTGATAGCAGTGCAACCCCTCTTTGGCCCACGGGCAGGAGGCACC TGTCTCACTCTTGAAGGCCAGAGTCTGTCTGTAGGCACCAGCCGGGCTGTGCTGGTCA ATGGGACTGAGTGTCTGCTAGCACG

\section{B.}

$<$ F2 sequence $>$ - $1000 \mathrm{bp}$

GTTTTCCAGGTACCTATCCAAGGCCCTGGCTGCCGCCACTTCCTGACCTGTGGGCGTT GCCTAAGGGCATGGCATTTCATGGGCTGTGGCTGGTGTGGGAACATGTGCGGCCAGC AGAAGGAGTGTCCTGGCTCCTGGCAACAGGACCACTGCCCACCTAAGCTTACTGAGTT CCACCCCCACAGTGGACCTCTAAGGGGCAGTACAAGGCTGACCCTGTGTGGCTCCAA CTTCTACCTTCACCCTTCTGGTCTGGTGCCTGAGGGAACCCATCAGGTCACTGTGGGC CAAAGTCCCTGCCGGCCACTGCCCAAGGACAGCTCAAAACTCAGgtacaatctggtccctcccct ccctttccctgaagggggaaaaccaagcagccccttccccatgagaccctgttctctgcttatcagaggcaaggggggatggggg aagctgcagtggttctgactgcttttgagagtcaaaaaggtctctgtcccttttgagcttcatgtg cccttcctgtctgttcactcatgg accagccaaggttaatctctgccccaccagaaccttccttccatggagggagg atgggtggagaaatgccattctctggctcag aggagccctgtggcttgtggcagg catgcatctaggcctgtgtaattcctggctgacctcaggggttcccctggtgccccagACC AGTGCCCCGGAAAGACTTTGTAGAGGAGTTTGAGTGTGAACTGGAGCCCTTGGGCAC CCAGGCAGTGGGGCCTACCAACGTCAGCCTCACCGTGACTAACATGCCACCGGGCAA GCACTTCCGGGTAGACGGCACCTCCGTGCTGAGAGGCTTCTCTTTCATGGAGCCAGT GCTGATAGCAGTGCAACCCCTCTTTGGCCCACGGGCAGGAGGCACCTGTCTCACTCTT GAAGGCCAGAGTCTGTCTGTAGGCACCAGCCGGGCTGTGCTGGTCAATGGGACTGAG TGTCTGCTAGCACG

Exon 4,5

Intron5

Exon6

Exon7

Figure 2. Sequence of the F1 and F2 fragment. (A) Partial sequences of the F1 fragment. F1 fragment contains exons 4 and 5, intron 5, exon 6, intron 6 and exon 7. (B) Partial sequences of the F2 fragment. F2 fragment contains exons 4 and 5, intron 5, exons 6 and 7 . The stop codon in intron 5 in frame with exons 1-4 is shown in bold.

with $10 \%$ of Fetal Bovine Serum (FBS) at $37^{\circ} \mathrm{C}$ in a humidified $5 \% \mathrm{CO}_{2}$ condition.

$R T-P C R$. Total RNA was extracted using RiboEx (GeneAll) following the manufacturer's instructions. Total RNA $(1 \mu \mathrm{g})$ was reverse transcribed with a RON exon 8 specific primer (5'-TGGCACATAAAAGCTG-3') using ImProm-II ${ }^{\mathrm{TM}}$ reverse transcriptase (Promega) following the manufacturer's instructions. RON cDNA was amplified by PCR using RON exon 4-specific primer (5'-GTTTTCCAGGTACCTATCCAAG-3') 


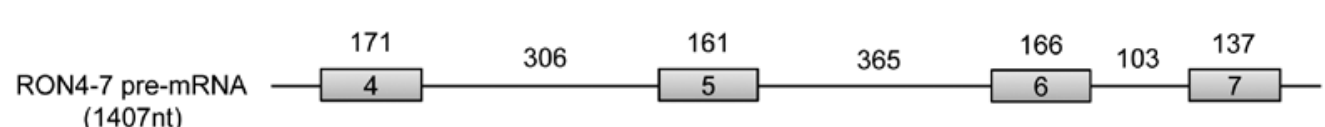

(1407nt)
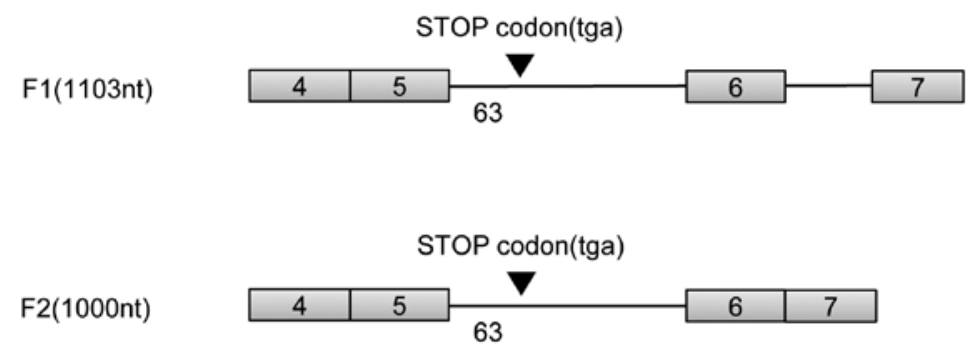

Figure 3. The schematic diagrams of the RON pre-mRNA, F1 andF2 fragments. Intron 4 is spliced in the F1 fragment while introns 4 and 6 , but not intron 5 , are spliced in F2 fragment through the alternative splicing. A premature stop codon in frame with exons 1 to exon 4 is located in intron 5 at $63 \mathrm{nt}$ downstream from the 5 ' splice site of exon 5 .

and RON exon 7 specific primer 5'-CGTGCTAGCAGACACT CAGTC-3'). The PCR products were loaded onto $2 \%$ agarose gel and visualized by staining with ethidium bromide solution. RT-PCR bands were purified and sequenced.

\section{Results}

Novel RT-PCR products are produced from RNA fragment covering exons 4-7. In the pre-mRNA splicing of RON gene, Ron $\Delta 160$ is produced due to skipping of exons 5 and 6 . In order to compare the skipping/inclusion of exons 5 and 6 in different cell lines, we performed RT-PCR reaction using primers from exons 4 and 7. As expected we observed several splicing variants that had been previously described to include or exclude exons 5 and 6 in human colon cancer HT-29 and HCT116 cells (23). In addition to these two isoforms, a variant which includes exon 6 , but not exon 5 , is also produced in these two cell lines, consistent with previous results (27). To our surprise, we also found that two other PCR products which we name as F1 and F2, are also produced, as shown in Fig. 1. As compared with size markers on the agarose gel, the length of $\mathrm{F} 1$ is $\sim 1,100 \mathrm{bp}$; the length of $\mathrm{F} 2$ is $\sim 1,000 \mathrm{bp}$. On the other hand, in cervix cancer cell C33A, none of any RT-PCR product RON RNA is produced; consistent with the fact that RON is not expressed in cervix cells. In HeLa cells, the only RT-PCR products we have observed are variants that have exon 5 and 6 inclusion. F1 and F2 products are not found in HeLa and C33A cells.

F1 and F2 fragments are partially spliced product of RON pre-mRNA. In order to characterize two unknown pre-mRNA splicing products from exons 4 and 7 of RON, we performed sequence analysis. As shown in Fig. 2, we found that the RNA fragment, which corresponds to F1 RT-PCR product, contains exons 4 and 5, intron 5, exon 6, intron 6 and exon 7 (1103Base). Another RNA fragment, which corresponds to F2 RT-PCR product, contains exons 4 and 5, intron 5, exons 6 and 7 (1000Base). Therefore F1 is a partial splicing product in which only intron 4 is spliced while F2 is a product in which both introns 4 and 6 are spliced as shown in Fig. 3. F1 and F2 intron
5 are not spliced in either $\mathrm{F} 1$ or $\mathrm{F} 2$ products. We conclude that F1 and F2 are partially spliced product of RON pre-mRNA.

Intron 5 includes a stop codon. Both novel RNA variants which correspond to F1 and F2 contain intron 5. We analyzed intron 5 sequence. We found that intron 5 contains a stop codon at the position 63-nt from 5' splice site of exon 5 in frame with exons 1-4. Therefore it is likely that F1 and F2 will encode truncated RON isoforms from exons 1-4, but not beyond exon 4 .

\section{Discussion}

The full length RON precursor is $180 \mathrm{kDa}$. But it has been welldocumented that RON has several isoforms that are produced from alternative splicing or protein truncation. Ron $\Delta 160$ without exons 5 and 6 is oncogenic while Ron $\Delta 170$ is a dominant negative isoform of RON protein. The different effects of these two isoforms suggest that it is important to investigate how many isoforms and what isoforms are present in particular cells. In this study, we used RT-PCR and identified two additional RON RNA variants both of which include intron 5 . The intron 5 sequence contains stop codons that prevent these two RON RNA variants to translate proteins beyond exon 4 , leading to potential truncated isoforms of RON protein (from exons 1-4). The existence and possible functions of these isoforms should be investigated in the future.

\section{Acknowledgements}

This study was supported by Mid-career Researcher Program through National Research Foundation (NRF) grant (20110000188 and 2011-0016757) funded by the Ministry of Education, Science, and Technology (MEST), Korea; a Systems Biology Infrastructure Establishment Grant provided by GIST in 2011.

\section{References}

1. Ronsin C, Muscatelli F, Mattei MG and Breathnach R: A novel putative receptor protein tyrosine kinase of the met family. Oncogene 8: 1195-1202, 1993. 
2. Trusolino L and Comoglio PM: Scatter-factor and semaphorin receptors: cell signalling for invasive growth. Nature reviews. Cancer 2: 289-300, 2002.

3. Wang MH, Wang D and Chen YQ: Oncogenic and invasive potentials of human macrophage-stimulating protein receptor, the RON receptor tyrosine kinase. Carcinogenesis 24: 1291-1300, 2003.

4. van der Geer P, Hunter T and Lindberg RA: Receptor proteintyrosine kinases and their signal transduction pathways. Annu Rev Cell Biol 10: 251-337, 1994.

5. Robertson SC, Tynan J and Donoghue DJ: RTK mutations and human syndromes: when good receptors turn bad. Trends Genet 16: 265-271, 2000 .

6. Birchmeier W, Brinkmann V, Niemann C, et al: Role of HGF/SF and c-Met in morphogenesis and metastasis of epithelial cells Ciba Found Symp 212: 230-240, 1997.

7. Niranjan B, Buluwela L, Yant J, et al: HGF/SF: a potent cytokine for mammary growth, morphogenesis and development. Development 121: 2897-2908, 1995

8. Vande Woude GF, Jeffers M, Cortner J, Alvord G, Tsarfaty I and Resau J: Met-HGF/SF: tumorigenesis, invasion and metastasis. Ciba Found Symp 212: 119-154, 1997.

9. Comoglio PM, Tamagnone L and Boccaccio C: Plasminogenrelated growth factor and semaphorin receptors: a gene superfamily controlling invasive growth. Exp Cell Res 253: 88-99, 1999.

10. Xu XM, Zhou YQ and Wang MH: Mechanisms of cytoplasmic $\{$ beta\}-catenin accumulation and its involvement in tumorigenic activities mediated by oncogenic splicing variant of the receptor originated from Nantes tyrosine kinase. J Biol Chem 280 25087-25094, 2005

11. Chen YQ, Zhou YQ, Angeloni D, Kurtz AL, Qiang XZ and Wang MH: Overexpression and activation of the RON receptor tyrosine kinase in a panel of human colorectal carcinoma cell lines. Exp Cell Res 261: 229-238, 2000.

12. Lu Y, Yao HP and Wang MH: Multiple variants of the RON receptor tyrosine kinase: biochemical properties, tumorigenic activities, and potential drug targets. Cancer Lett 257: 157-164, 2007.

13. Gaudino G, Follenzi A, Naldini L, et al: RON is a heterodimeric tyrosine kinase receptor activated by the HGF homologue MSP EMBO J 13: 3524-3532, 1994.

14. Skeel A, Yoshimura T, Showalter SD, Tanaka S, Appella E and Leonard EJ: Macrophage stimulating protein: purification, partial amino acid sequence, and cellular activity. J Exp Med 173: 1227-1234, 1991
15. Bezerra JA, Witte DP, Aronow BJ and Degen SJ: Hepatocytespecific expression of the mouse hepatocyte growth factor-like protein. Hepatology 18: 394-399, 1993.

16. Danilkovitch A and Leonard EJ: Kinases involved in MSP/RON signaling. J Leukoc Biol 65: 345-348, 1999.

17. Danilkovitch-Miagkova A and Zbar B: Dysregulation of Met receptor tyrosine kinase activity in invasive tumors. J Clin Invest 109: 863-867, 2002.

18. Wang MH, Montero-Julian FA, Dauny I and Leonard EJ Requirement of phosphatidylinositol-3 kinase for epithelial cell migration activated by human macrophage stimulating protein. Oncogene 13: 2167-2175, 1996.

19. Santoro MM, Collesi C, Grisendi S, Gaudino G and Comoglio PM: Constitutive activation of the RON gene promotes invasive growth but not transformation. Mol Cell Biol 16: 7072-7083, 1996.

20. Camp ER, Liu W, Fan F, Yang A, Somcio R and Ellis LM: RON, a tyrosine kinase receptor involved in tumor progression and metastasis. Ann Surg Oncol 12: 273-281, 2005.

21. Rodrigues GA and Park M: Oncogenic activation of tyrosine kinases. Curr Opin Genet Dev 4: 15-24, 1994.

22. Wang MH, Lee W, Luo YL, Weis MT and Yao HP: Altered expression of the RON receptor tyrosine kinase in various epithelial cancers and its contribution to tumourigenic phenotypes in thyroid cancer cells. J Pathol 213: 402-411, 2007.

23. Wang MH, Kurtz AL and Chen Y: Identification of a novel splicing product of the RON receptor tyrosine kinase in human colorectal carcinoma cells. Carcinogenesis 21: 1507-1512, 2000.

24. Wang MH, Lao WF, Wang D, Luo YL and Yao HP: Blocking tumorigenic activities of colorectal cancer cells by a splicing RON receptor variant defective in the tyrosine kinase domain. Cancer Biol Ther 6: 1121-1129, 2007.

25. Collesi C, Santoro MM, Gaudino G and Comoglio PM: A splicing variant of the RON transcript induces constitutive tyrosine kinase activity and an invasive phenotype. Mol Cell Biol 16: 5518-5526, 1996.

26. Zhou YQ, He C, Chen YQ, Wang D and Wang MH: Altered expression of the RON receptor tyrosine kinase in primary human colorectal adenocarcinomas: generation of different splicing RON variants and their oncogenic potential. Oncogene 22: 186-197, 2003.

27. Eckerich C, Schulte A, Martens T, Zapf S, Westphal M and Lamszus K: RON receptor tyrosine kinase in human gliomas: expression, function, and identification of a novel soluble splice variant. J Neurochem 109: 969-980, 2009. 